\title{
Nucleation from a cluster of inclusions, leading to void coalescense
}

\section{Tvergaard, Viggo}

\section{Published in:}

International Journal of Mechanical Sciences

Link to article, DOI:

10.1016/j.ijmecsci.2017.09.027

Publication date:

2017

Document Version

Peer reviewed version

Link back to DTU Orbit

\section{Citation (APA):}

Tvergaard, V. (2017). Nucleation from a cluster of inclusions, leading to void coalescense. International Journal of Mechanical Sciences, 133, 631-638. https://doi.org/10.1016/j.ijmecsci.2017.09.027

\section{General rights}

Copyright and moral rights for the publications made accessible in the public portal are retained by the authors and/or other copyright owners and it is a condition of accessing publications that users recognise and abide by the legal requirements associated with these rights.

- Users may download and print one copy of any publication from the public portal for the purpose of private study or research.

- You may not further distribute the material or use it for any profit-making activity or commercial gain

- You may freely distribute the URL identifying the publication in the public portal

If you believe that this document breaches copyright please contact us providing details, and we will remove access to the work immediately and investigate your claim 


\title{
NUCLEATION FROM A CLUSTER OF INCLUSIONS, LEADING TO VOID
}

\section{COALESCENSE}

\author{
Viggo Tvergaard \\ Department of Mechanical Engineering, Solid Mechanics \\ Technical University of Denmark, DK-2800 Kgs. Lyngby, Denmark
}

\begin{abstract}
A cell model analysis is used to study the nucleation and subsequent growth of voids from a nonuniform distribution of inclusions in a ductile material. Nucleation is modeled as either stress controlled or strain controlled. The special clusters considered consist of a number of uniformly spaced inclusions located along a plane perpendicular to the maximum principal tensile stress. A plane strain approximation is used, where the inclusions are parallel cylinders perpendicular to the plane. Clusters with different numbers of inclusions are compared with the nucleation and growth from a single inclusion, such that the total initial volume of the inclusions is the same for the clusters and the single inclusion. After nucleation, local void coalescence inside the clusters is accounted for, since this makes it possible to compare the rate of growth of the single larger void that results from coalescence in the different clusters. Nucleation parameters leading to rather early nucleation, or to later nucleation, are considered. Also, different transverse stresses on the unit cell are considered to see the influence of different levels of stress triaxiality, and results are shown for different levels of strain hardening in the material.
\end{abstract}

KEYWORDS: Void cluster, nucleation, large strain plasticity, ductile fracture.

\section{INTRODUCTION}

Early models for void growth in ductile metals under tensile loading have been developed by McClintock [1] and Rice and Tracey [2], and subsequently a large amount of research has focussed on this area (see reviews by Garrison and Moody [3]; Tvergaard [4]; Benzerga and Leblond [5]; Benzerga et al. [6]). Most models for ductile porous materials have considered solids with uniformly distributed voids, but in real materials the voids are often more or less randomly distributed, so that the effect of non-uniform distances between voids can be of importance to the material response. This has been illustrated by Becker [7] for specimens made of partially consolidated and sintered iron powder, with analyses based on the constitutive equations of Gurson [8] extended in (Tvergaard [9]; Tvergaard and Needleman [10]).

To study non-uniform void distributions, Geltmacher et al. [11] have considered thin metal sheets under either uniaxial or equal-biaxial tension, with various random distributions of through-thickness holes. Thomson et al. [12,13] have studied periodic clusters of particles 
in the form of two closely spaced spherical particles, placed either on a line perpendicular to the maximum principal stress direction, or on an inclined line, with void nucleation modelled by a cohesive zone at the interface, or by failure in a secondary population of small voids represented by the Gurson model. The two voids on a line perpendicular to the maximum principal stress direction were found to be most critical. Bandstra and Koss [14] have analysed a cluster of three closely spaced spherical voids, by considering a 3D finite element solution for a triangular material region that contains only part of one void. Babout et al. [15] have experimentally studied a model material fabricated by powder metallurgy with spherical ceramic particles in either commercially pure aluminium, or in a hardening aluminium alloy. For such a material Segurado and LLorca [16] have made 3D finite element studies, accounting for different clusters of spherical particles within the unit cell. A disk shaped cluster normal to the maximum tensile stress direction has also been studied by Ohno and Hutchinson [17] in an axisymmetric solution, with the cluster represented by a Gurson material, to determine when the effect of the cluster is strong enough to give plastic flow localization in the band containing the cluster.

With random void distributions in front of a crack-tip, represented in terms of a Gurson material, it has been found (Tvergaard and Needleman [18,19]) that first hole linking is promoted by a decreased interhole spacing as occurs in clusters of voids. Also, experimental studies for a resulferised stainless steel (AISI 303) have shown (Tinet et al. [20]) that small micro-cracks can develop by local coalescence well before the final catastrophic void coalescence.

A previous study of cluster effects (Tvergaard [21]) has considered the interaction of a number of voids located on a line perpendicular to the maximum principal stress direction, with the purpose of comparing the effect of clusters with different numbers of voids, but with the same total initial void volume fraction. This study was carried out in a plane strain context so that the voids are actually parallel circular cylindrical voids, and an important aspect of the analyses was that void coalescence inside the cluster is accounted for, so that the cluster of small voids finally develops into a single larger void. In the present paper this study is extended by considering similar clusters of inclusions and studying the effect of nucleation. Both a strain controlled nucleation mechanism and a stress controlled mechanism are modelled. 
The cluster of inclusions to be analysed has the same initial geometry as the voids analysed in (Tvergaard [21]). Thus, the inclusions are located at the center of a rectangular region with the initial width $2 A_{0}$ in the $x^{1}$-direction and the initial height $2 B_{0}$ in the $x^{2}$-direction, the initial inclusion radius is $R_{0}$ and the number of inclusions in the cluster is denoted $N_{v}$, with the inclusions aligned on the $x^{1}$-axis, as illustrated in Fig. 1a. Due to symmetries only one quarter of the region is analysed, as shown with the dimensions $A_{0} \times B_{0}$ in Fig. 1b. The case of Fig. 1a corresponds to $N_{v}=5$, where only half of the inclusions in the cluster are visible due to the assumed symmetry.

The inclusions are modelled as rigid, free to move along the $x^{1}$-axis so that the resulting force on each inclusion in the $x^{1}$-direction is zero. The matrix material is taken to be perfectly bonded to the inclusions initially and nucleation is taken to occur either by a stress controlled mechanism or by a plastic strain controlled mechanism. Stress controlled nucleation is taken to occur when the average stress $\Sigma_{n}$ on the centre line in the inclusion, in the $x^{2}$-direction, reaches a critical value $\Sigma_{N}$. Here $\Sigma_{n}$ is defined as

$$
\Sigma_{n}=\frac{1}{2 R_{0}} \int_{S_{I}} T^{2} d s
$$

where $S_{I}$ is the length of the inclusion surface in the part with $x^{2}>0$, the parameter $s$ measures length along that surface, and $T^{2}$ is the nominal traction component in the $x^{2}$ direction on the inclusion surface. Plastic strain controlled nucleation is taken to occur if the average $\varepsilon_{n}$ of the plastic strain along the surface of the inclusion reaches a critical value $\varepsilon_{N}$. Here $\varepsilon_{n}$ is defined by

$$
\varepsilon_{n}=\frac{1}{S_{I}} \int_{S_{I}} \varepsilon^{p} d s
$$

where $\varepsilon^{p}$ is the effective plastic strain in the metal along the inclusion surface. For each inclusion both the stress controlled condition and the strain controlled condition are checked throughout the analysis, prior to nucleation for that inclusion. When one of the conditions is satisfied nucleation is started at that inclusion, such that all nodes along the inclusion are released and the nodal forces are stepped down in a number of subsequent increments.

It is noted that the nucleation process could also have been described by using a cohesive zone model, as has been done for inclusions (Needleman [22]) or short fibres (Tvergaard [23]), specifying a stress controlled nucleation. Then separation along the inclusion interface 
would start locally at the point of the inclusion surface where the stress is highest, and subsequently spread along the interface. However, the method of letting the averages (1) and (2) determine the onset of nucleation at each inclusion is found sufficient here, to get an impression of the effect of differences in the nucleation stress or the nucleation strain. The main issue is not the gradual separation of an inclusion but the delay in void growth due to accounting for nucleation and the sequence of nucleation due to the non-uniform stress and strain state around the cluster of inclusions studied.

A convected coordinate Lagrangian formulation of the field equations is used for the analyses, with the Cartesian $x^{i}$ coordinate system as reference and with the displacement components on reference base vectors denoted by $u^{i}$. The metric tensors in the reference configuration and the current configuration, respectively, are $g_{i j}$ and $G_{i j}$ with determinants $g$ and $G$. In terms of the displacement components the Lagrangian strain tensor is

$$
\eta_{i j}=\frac{1}{2}\left(u_{i, j}+u_{j, i}+u_{, i}^{k} u_{k, j}\right)
$$

where ()$_{, j}$ denotes covariant differentiation in the reference frame. The contravariant components $\tau^{i j}$ of the Kirchhoff stress tensor on the current base vectors are related to the components of the Cauchy stress tensor $\sigma^{i j}$ by $\tau^{i j}=\sqrt{G / g} \sigma^{i j}$. For the finite strain formulation of the $J_{2}$ flow theory applied, with the Mises yield surface, the incremental stress-strain relationship takes the form $\dot{\tau}^{i j}=L^{i j k \ell} \dot{\eta}_{k \ell}$, with the instantaneous moduli specified in (Hutchinson [24]; Tvergaard [25]). With Young's modulus $E$, the initial yield stress $\sigma_{Y}$ and the power hardening exponent $n$, the true stress-logarithmic strain curve in uniaxial tension is taken to follow the power law

$$
\varepsilon= \begin{cases}\sigma / E, & \sigma \leq \sigma_{Y} \\ \left(\sigma_{Y} / E\right)\left(\sigma / \sigma_{Y}\right)^{n}, & \sigma \geq \sigma_{Y}\end{cases}
$$

In the plane strain unit cell analysis, where $N_{v}$ is the number of inclusions in the cluster, the initial inclusion volume fraction is

$$
f_{I}=N_{v} \pi R_{0}^{2} /\left(4 A_{0} B_{0}\right)
$$

The different clusters to be compared here have the same value of the initial inclusion volume fraction $f_{I}$, but different values of $N_{v}$. Thus, for a fixed value of $f_{I}$ the initial void radius $R_{0}$ is different for each cluster. The behaviour to be compared involves the onset of 
nucleation at the different inclusions, the subsequent growth of the voids, the coalescence and finally the growth of coalesced voids.

On the four sides of the quarter unit cell (Fig. 1b) symmetry boundary conditions are applied, so that the sides are kept straight. The two sides located on the coordinate axes are prescribed to remain on the axes, with the incremental boundary conditions

$$
\begin{array}{lllll}
\Delta u_{1}=0 & \text { and } & \Delta T^{2}=0 & \text { at } & x^{1}=0 \\
\Delta u_{2}=0 & \text { and } & \Delta T^{1}=0 & \text { at } & x^{2}=0
\end{array}
$$

where $\Delta T^{i}$ denotes the nominal traction increments. The other two sides have prescribed displacements $U_{1}$ or $U_{2}$ in the directions normal to the sides, with the incremental boundary conditions

$$
\begin{array}{lllll}
\Delta u_{1}=\Delta U_{1} & \text { and } & \Delta T^{2}=0 & \text { at } & x^{1}=A_{0} \\
\Delta u_{2}=\Delta U_{2} & \text { and } & \Delta T^{1}=0 & \text { at } & x^{2}=B_{0}
\end{array}
$$

The average logarithmic strains in the two coordinate directions are $\varepsilon_{1}=\ln \left(1+U_{1} / A_{0}\right)$ and $\varepsilon_{2}=\ln \left(1+U_{2} / B_{0}\right)$, while the average true stresses $\Sigma_{11}$ and $\Sigma_{22}$ in the two coordinate directions are the averages of the stresses on the relevant edge of the deformed unit cell. In each increment of the solution the displacement increment $\Delta U_{2}$ is prescribed, while the increment $\Delta U_{1}$ is calculated such that the ratio of the macroscopic stresses remains fixed

$$
\Sigma_{11} / \Sigma_{22}=\kappa
$$

By prescribing different values of the constant $\kappa$ the nucleation of voids at the inclusions and the subsequent void growth can be compared at different levels of stress triaxiality.

When neighbouring voids grow large enough to interact coalescence will develop. This process is here approximated by releasing the forces in the ligament when it has become so thin, that the ratio of the current ligament thickness to the initial ligament thickness has reached a critical value $\chi_{c}$. At this point the nodal forces on the ligament are stepped down to zero during a number of subsequent increments. Here, the value of $\chi_{c}$ is taken to be 0.3 . It is noted that different values of $\chi_{c}$ have been used in earlier analyses of crack growth through a row of discrete voids, 0.6 in (Tvergaard and Hutchinson [26]) and 0.15 in 
(Tvergaard [27]). In very pure metals necking sometimes continues down to a point so that a very small value of $\chi_{c}$ would be appropriate, but in many structural alloys the neck typically breaks before that. Here the value $\chi_{c}=0.3$ is chosen as a reasonable approximation to such cases where the ligament breaks before it has necked down to a point.

\section{NUMERICAL SOLUTION}

The principle of virtual work expanded about the current state takes the form

$$
\int_{V}\left\{\Delta \tau^{i j} \delta \eta_{i j}+\tau^{i j} \Delta u_{, i}^{k} \delta u_{k, j}\right\} d V=\int_{A} \Delta T^{i} \delta u_{i} d A-\left[\int_{V} \tau^{i j} \delta \eta_{i j} d V-\int_{A} T^{i} \delta u_{i} d A\right]
$$

Here, $V$ and $A$ are the volume and surface of the body in the reference configuration, $\Delta \tau^{i j}$ and $\Delta \eta_{i j}$ are the stress and strain increments, $T^{i}$ are contravariant components of the nominal surface tractions. The bracketed terms are equilibrium corrections. The displacement fields are approximated in terms of 8-noded isoparametric elements, with the volume integral in (11) carried out by using $2 \times 2$ point Gauss integration within each element. Fig. 2 illustrates the mesh near the inclusions for $N_{v}=9$. In each increment of the finite element solution the boundary conditions (6)-(9) are applied. Before nucleation the matrix material is taken to be perfectly bonded to the inclusion, then at nucleation the nodes on the inclusion interface are released and the corresponding nodal forces are stepped down during a number of increments. Subsequently, the nominal tractions on the void surface are taken to be zero. In the nucleation conditions, the integral in (1) is calculated as the sum of the nodal force components in the $x^{2}$-direction on the matrix-inclusion interface, and the effective plastic strain in (2) is calculated as the average in each of the elements adjacent to the interface. When a ligament between voids reaches the critical thinning so that coalescence initiates, the boundary condition (7) is relaxed at that ligament and the corresponding nodal forces are stepped down to zero during the following increments.

In each increment a special Rayleigh-Ritz finite element method (Tvergaard [25]) is used to maintain the prescribed stress ratio (10). This method also makes it possible to prescribe internal displacement increments in the material above voids without applying a force there, to be able to continue the solution through unstable equilibria that occur during the node force release procedures used to represent both the void nucleation and the coalescence process. 
Also, prior to nucleation, the displacements of a rigid inclusions in the $x^{1}$-direction are introduced as extra variables, to enforce zero forces on the inclusions in the $x^{1}$-direction.

During the coalescense severe mesh distortion tends to occur, which is avoided by using a remeshing procedure developed by Pedersen [28] and Tvergaard [29,30]. The values of field quantities in the integration points of the new mesh are determined by interpolation in the old mesh. A remeshing is carried out when $\Delta \varepsilon_{e} \geq\left(\Delta \varepsilon_{e}\right)_{\max }$ in any integration point, where $\varepsilon_{e}=\int\left(2 \dot{\eta}_{i j} \dot{\eta}^{i j} / 3\right)^{1 / 2} d t$ is an effective strain measure, and $\Delta \varepsilon_{e}$ denotes the amount of this strain accumulated since last remeshing. Here, the limiting value $\left(\Delta \varepsilon_{e}\right)_{\max }$ is mostly chosen as 0.4 .

\section{RESULTS}

The material parameters in the analyses are taken to be $\sigma_{Y} / E=0.003, v=1 / 3$ and in most cases $n=10$. The initial geometry of the cell model and the cluster are taken to equal those used in [21], so that $A_{0}=B_{0}$ and the initial inclusion volume fraction is $f_{I}=0.1295 * 10^{-5}$. Thus, for $N_{v}=1$ the initial inclusion radius is $R_{0}=0.001286 A_{0}$, for $N_{v}=5$ it is $R_{0}=0.000575 A_{0}$ and for $N_{v}=9$ it is $R_{0}=0.0004286 A_{0}$. The initial spacings between inclusions are taken to be $X_{0}=0.02143 A_{0}$.

In the first case analysed the ratio of the transverse stress to the main tensile stress is taken to be given by $\kappa=0.25$ in Eq. (10). This corresponds to a macroscopic stress triaxiality, mean stress divided by Mises stress, of about 0.96 . Even though the macroscopic principal stress $\Sigma_{33}$ is not calculated here, an assumption of zero plastic strain rate and thus zero stress deviator in the $x^{3}$-direction would result in $\Sigma_{33}=\Sigma_{22}(1+\kappa) / 2$. This stress value is in good agreement with stresses computed in the field well away from the voids, and this gives the stress triaxiality 0.96 .

The condition for stress controlled void nucleation at the inclusions is here given by the value $\Sigma_{N} / \sigma_{Y}=3.33$, specifying the critical value of the stress measure in (1). The condition for strain controlled nucleation is given by the critical value $\varepsilon_{N}=0.1$ of the strain measure in (2). For the three different values of $N_{v}$ considered Fig. 3a shows the variation of the 
normalised tensile stress $\Sigma_{22} / \sigma_{Y}$ vs. the tensile logarithmic strain $\varepsilon_{2}$ and Fig. 3b shows the corresponding evolution of the total volume $V$ of inclusions or voids, normalised by the initial inclusion volume $V_{0}$. In this case, for each of the three values of $N_{v}$, all the inclusions reach the critical strain before the critical stress, so that strain controlled nucleation dominates. All these nucleations take place within a narrow range of the strain $\varepsilon_{2}$, between 0.093 and 0.104, indicated by the sharp load drop during the nucleation process in Fig. 3a. Prior to the nucleation there has been no void growth, but even after the nucleation there is very slow void growth only visible at much larger strain in Fig. 3b. Also the ligament failures happen within a narrow range of strain, around $\varepsilon_{2}=0.211$ for $N_{v}=5$ and $\varepsilon_{2}=0.231$ for $N_{v}=9$. Both these strain values are increased by about 0.03 , from 0.175 and 0.199 , respectively, relative to the values found in [21] for the same case with the voids present from the beginning. While the ligament failure is being modelled by stepping down the nodal forces on the ligament, the stress drops significantly, analogous to the behaviour seen during void nucleation. In an experiment under displacement control there would instead be some local dynamics, as the solution would snap.

The behaviour found in Figs. 3a and $3 b$ is similar to that determined in [21] for voids present from the beginning, except for the delay in strain by about about 0.03 , regarding the ligament breakage and the initiation of rapid void growth. Thus, the ligaments break earlier in the cluster with $N_{v}=5$ than in the cluster with more voids, but when the ligaments break in the cluster with $N_{v}=9$ the total void volume grows more rapidly. Also, after void coalescence the total void volume in both clusters grows faster than that of the single void. It is noted that when $V / V_{0}$ reaches the big value 1000 the void volume fraction $f$ is still only about 0.00129 because the initial inclusion volume fraction in the cases analysed is very small. The volume change of the unit cell is small, as it results only from elastic dilatation and from the growth of a very small void volume fraction.

A deformed mesh at the end of the computation with $N_{v}=9$ in Fig. 3 is shown in Fig. 4, corresponding to the initial mesh shown in Fig. 2. Here the value of the axial strain is $\varepsilon_{2}=0.255$ and the void volume $V / V_{0}=1197$. The material around the broken ligaments has hardly deformed since the occurrence of coalescence, but it is seen that the ligament closest to the centre has not opened quite as much as the outer ligament, because the outer ligament breaks first. It is noted that the edge of the big void in Fig. 4 develops into a shape where it 
forms an angle with the $x^{1}$-axis, even though all voids start from the circular cylindrical shapes of the inclusions. The mesh around the smaller voids, which have coalesced, shows that also here the edges have developed an angle to the $x^{1}$-axis before the ligaments failed. Part of the reason for this is elastic unloading that takes place at the top of the voids.

In the case of Fig. 5 the critical stress for nucleation is somewhat reduced relative to Fig. 3 , to the value $\Sigma_{N} / \sigma_{Y}=2.33$, while the critical strain value is unchanged, at $\varepsilon_{N}=0.1$. This results in stress controlled nucleation at a smaller strain than found in Fig. 3, at $\varepsilon_{2}=0.080$ for $N_{v}=1$ and in the range 0.082 to 0.085 for $N_{v}=5$. In the case of $N_{v}=9$ the two nucleation conditions compete, first nucleation occurs at the outer inclusion by the strain condition, at $\varepsilon_{2}=0.093$ as in Fig. 3, and most other inclusions also reach the strain condition first, but the central inclusion nucleates by the stress condition at $\varepsilon_{2}=0.096$, which means that the last nucleation occurs earlier than in Fig. 3, at $\varepsilon_{2}=0.097$. Due to the earlier occurrence of nucleation also the ligament breakage and the final high values of the total void volume are reached a little earlier than found in Fig. 3.

In Fig. 6 the critical stress for nucleation is kept at the relatively low value $\Sigma_{N} / \sigma_{Y}=2.33$, also considered in Fig. 5, but the critical strain for nucleation is increased to $\varepsilon_{N}=0.15$, higher than the value considered in Figs. 3 and 5. As expected this results in stress controlled nucleation at all inclusions considered, and the curves for $N_{v}=1$ and $N_{v}=5$ are identical to those in Fig. 5, since also here stress controlled nucleation happened. However, for $N_{v}=9$ the higher value of $\varepsilon_{N}$ suppresses strain controlled nucleation, so that nucleation occurs somewhat later when the critical value of the stress measure (1) is reached. This means that nucleation occurs later at values of $\varepsilon_{2}$ in the range 0.124 to 0.127 and that also ligament failure is delayed to values of $\varepsilon_{2}$ in the range 0.251 to 0.252 .

The effect of the stress triaxiality is investigated in the next two figures, by prescribing $\kappa=0.40$ in Fig. 7, which corresponds to a stress triaxiality of about 1.35 , and $\kappa=0.0$ in Fig. 8, which corresponds to a stress triaxiality of about 0.58 . The nucleation parameters are taken to be $\Sigma_{N} / \sigma_{Y}=3.33$ and $\varepsilon_{N}=0.1$, as in Fig. 3. For the higher stress triaxiality in Fig. 7 the stress level becomes higher, but all nucleations still occur by the strain controlled mechanism, at values of $\varepsilon_{2}$ in the range 0.098 to 0.106 , close to the range found in Fig. 3 . However, after nucleation the higher stress level gives faster void growth so that the 
ligaments break at significantly smaller strains, around $\varepsilon_{2}=0.147$ for $N_{v}=5$ and $\varepsilon_{2}=0.158$ for $N_{v}=9$. In Fig. 8, note that the horizontal axis is stretched to allow for the larger strains reached. Also here, at the lower stress triaxiality, all nucleations occur by the strain controlled mechanism, at values of $\varepsilon_{2}$ in the range 0.095 to 0.098 , still close to the ranges found in Figs. 3 and 7. But after nucleation the voids grow much more slowly due to the lower stress level in the material. Therefore the ligaments break at significantly larger strains, around $\varepsilon_{2}=0.425$ for $N_{v}=5$ and $\varepsilon_{2}=0.471$ for $N_{v}=9$. The growth of the total void volume in Figs. $7 \mathrm{~b}$ and $8 \mathrm{~b}$ follows the same trend as found in Fig. 3b, that noticeable growth starts earlier for $N_{v}=1$, but when the ligaments break in the clusters their total void volume grows faster than that of the single void, and the volume grows faster for the cluster with $N_{v}=9$ than for the cluster with only 5 inclusions.

In Fig. 9 nucleation occurs much later, as both nucleation parameters are higher. The condition for stress controlled void nucleation is here given by the value $\Sigma_{N} / \sigma_{Y}=6.67$, while the condition for strain controlled nucleation is given by $\varepsilon_{N}=0.3$. The stress triaxiality level is specified by prescribing the stress ratio $\kappa=0.25$, as in Figs. 3, 5 and 6 . The higher critical stress for nucleation is not reached in any of the cases in Fig. 9, so all nucleation occurs when the increased nucleation strain $\varepsilon_{N}=0.3$ is reached. For the three values of $N_{v}$ practically all nucleation occurs at about the same values of the strain $\varepsilon_{2}$, around 0.29 , except for the central inclusion in the case of $N_{v}=9$, which nucleates at $\varepsilon_{2}=0.37$. In the case of $N_{v}=9$ the outer ligament breaks first at $\varepsilon_{2}=0.366$, just before nucleation at the central inclusion, and the other ligaments break soon after, at $\varepsilon_{2}=0.37$ nearly simultaneous with the last nucleation. For $N_{v}=5$ the ligaments break earlier than found for the larger number if inclusions, at $\varepsilon_{2}=0.348$, and therefore the total void volume starts to grow large at this strain, earlier than the rapid growth found for $N_{v}=9$.

Fig. 10 shows the deformed mesh at the end of the computation with $N_{v}=9$ in Fig. 9. Here the value of the axial strain is $\varepsilon_{2}=0.395$ with the total void volume $V / V_{0}=1197$. A main difference from the deformed state in Fig. 4 is that in Fig. 10 the material around the void is much more deformed. Also, it is clearly seen that the outer ligament broke first, as the opening between broken ligaments is larger. The remaining part of the central void shows that 
this void has grown less than the others, which follows from the fact that the central inclusion nucleated later.

Figs. 11 and 12 illustrate the influence of the strain hardening exponent on nucleation and subsequent void growth at the cluster of inclusions. In both cases the stress triaxiality level is specified by prescribing $\kappa=0.25$, as in Figs. 3, 5, 6 and 9, and the nucleation parameters are taken to be $\Sigma_{N} / \sigma_{Y}=3.33$ and $\varepsilon_{N}=0.1$, as in Figs. 3, 7 and 8 . The material in Fig. 11 is more high hardening, with the power hardening exponent $n=5$. This tends to give a higher stress level at a given strain, and therefore all nucleation in Fig. 11 occurs by the stress controlled criterion. For $N_{v}=9$ nucleation occurs near the strain $\varepsilon_{2}=0.065$ and the ligaments break close to the strain $\varepsilon_{2}=0.286$. For $N_{v}=5$ nucleation occurs a little later, around $\varepsilon_{2}=0.068$, but the ligaments break earlier, around $\varepsilon_{2}=0.266$. Thus nucleation occurs at a smaller strain than found in Fig. 3, but the ligaments break at larger strains.

The material in Fig. 12 is relatively low hardening, with the power hardening exponent $n=20$. Here the stresses at a given strain tend to be lower, so most nucleations occur by the strain controlled criterion, at $\varepsilon_{2}=0.104$ for $N_{v}=1$ and at strains between 0.098 and 0.108 for $N_{v}=9$, where the subsequent ligament failures occur in the narrow strain range between 0.194 and 0.197 . However, for $N_{v}=5$ only the outer inclusions nucleate by the strain controlled mechanism, at strains of 0.100 and 0.109 , respectively, while a shielding effect keeps the strain at the central inclusion below the critical value. At $\varepsilon_{2}=0.178$ the outer ligament breaks, and immediately after nucleation at the central inclusion occurs by the stress controlled mechanism. Then, soon after, at $\varepsilon_{2}=0.180$, the last ligament breaks. Thus, compared to the results in Fig. 3, the trends are opposite to those found in Fig. 11, the first nucleations occur at slightly larger strains, but the ligaments break at smaller strains.

\section{DISCUSSION}

The nucleation criteria used here are based on an average normal stress (1) on the inclusion, or on an average plastic strain (2) along the surface of the inclusion. Stress controlled nucleation could also be directly specified in terms of a cohesive zone around the particle, as has been done in [31] for an inclusion or in [32] for a whisker reinforced metal. A strain dependence of the nucleation could be included in a cohesive zone model, following the 
procedure suggested in [33]. However, the simplified procedure applied here is expected to give a good indication of the effect of stress or strain on nucleation of voids at the inclusions.

As in the previous investigation of clusters of pre-existing voids [21] the focus here is on comparing different clusters of inclusions that all have the same initial inclusion volume fraction, to see which of the clusters is most damaging when all the voids have coalesced into one. Also here the clusters compared have the inclusions located along a line perpendicular to the largest principal tensile stress. As in the previous study it turns out that the clusters of either 5 or 9 equally spaced inclusions lead to more rapid void growth in the final stage, after nucleation and coalescence of the voids, than found for the single inclusion. But the final void at the cluster grows large at a later stage when void growth is delayed by late occurrence of nucleation.

The abrupt load drop at nucleation occurs due to the redistribution of the stress field as the nodes on the matrix-inclusion interface are released and the corresponding nodal forces are stepped down. Here the solution is taken through an unstable equilibrium, where both the overall stress and the overall strain decay, while the opening at the matrix-inclusion interface increases. In an experiment with increasing strain prescribed such unstable equilibrium would be replaced by a dynamic snap.

If the cluster contained inclusions above those on the $x^{1}$-axis considered here, interaction between voids would most likely involve intense shearing. However, as the focus here is on clusters with a fixed total volume fraction of inclusions, the size of the voids would be smaller if a different distribution involved more voids in a cluster.

It is noted that the 3D study in [10] also accounts for nucleation and subsequent void growth, but in a manner quite different from that applied here. In [10] all is represented in terms of the Gurson model, such that a population of larger inclusions is represented as islands of stress controlled nucleation that tend to give nucleation at rather small strains, while a second population of inclusions in the matrix material between the larger inclusions are taken to nucleate voids at larger strains. Thereby, coalescence of the larger voids can develop by the formation of plastic flow localization in the matrix material between them, with subsequent failure in the band representing coalescence of the small scale voids. In the present study there is only one population of inclusions in the cluster, and coalescence is represented by an approximate model of the ligament necking.

It is noted that the computations here are not continued to consider final failure of the materials represented by the unit cells, which would involve interaction between voids in 
neighbouring unit cells. Even though the void volumes grow by a huge factor relative to the initial inclusion volumes, the void volume fractions at the end of the computations are still low, around $f=0.015$, and the spacing between these resulting voids is still much larger than the void size.

The very small total inclusion volume fraction has been chosen here to avoid noticeable interaction between neighbouring clusters. If this also involved very small voids, with diameters comparable to a characteristic length scale for the material, the rate of void growth would be reduced by strain gradient effects. However, the analyses here do not account for non-local effects.

The studies with a higher or lower strain hardening clearly show that the strain hardening affects the onset of nucleation and also the subsequent void growth. A more high hardening material tends to give higher stresses that promote earlier void nucleation, but the subsequent void growth is slower. On the other hand, a low hardening material gives lower stress levels, which tends to delay void nucleation, but the subsequent void growth is faster.

The choice of the critical value $\chi_{c}$ for ligament thinning also has an influence on the failure predictions. Here the value 0.3 was chosen, but other values have been tried in studies of crack growth. If a smaller value was chosen, to better model necking of the ligament down to a point, the predicted strain at which the voids in a cluster coalesce to a single void would be slightly increased.

The cases of increased or reduced stress triaxiality studied here did not have much influence on the onset of nucleation, because in those cases nucleation was strain controlled. The higher stress triaxiality gives higher stress levels and therefore, if stress controlled nucleation was first critical it would occur earlier for the higher stress triaxiality. After nucleation the voids grow much faster at the higher stress triaxiality, as expected.

The question studied by Ohno and Hutchinson [17], whether or not the cluster would result in plastic flow localization in the band along the $x^{1}$-axis, containing the cluster, has not been studied here. It would be possible with the present type of analysis, but would require a fine mesh all along the $x^{1}$-axis. However, by comparing with the void volume fractions considered in [15], even the local void volume fraction at the cluster in a band of width equal to the initial void spacing, there is no indication that localization would happen in this case, where the material outside the cluster is void free. 
Acknowledgements. The study is partially supported by the Technical University of Denmark. 


\section{REFERENCES}

1. McClintock, F.A. A criterion for ductile fracture by growth of holes. J. Appl. Mech., 1968, 35:363-371.

2. Rice, J.R. and Tracey, D.M. On the ductile enlargement of voids in triaxial stress fields. J. Mech. Phys. Solids, 1969, 17:201-217.

3. Garrison Jr, W.M., Moody, N.R. Ductile fracture. J. Phys. Chem. Solids, 1987, 48(11):1035-1074.

4. Tvergaard, V. Material failure by void growth to coalescence. Advances in Applied Mechanics, 1990, 27:83-151.

5. Benzerga, A.A., Leblond, J.-B. Ductile fracture by void growth to coalescence. Advances in Applied Mechanics, 2010, 44:169-305.

6. Benzerga, A.A., Leblond, J.-B., Needleman, A., Tvergaard, V. Ductile failure modelling. Int. J. Fracture, 2016, 201:29-80.

7. Becker, R. The effect of porosity distribution on ductile failure. J. Mech. Phys. Solids, 1987, 35:577-599.

8. Gurson, A.L. Continuum theory of ductile rupture by void nucleation and growth $-\mathrm{I}$. Yield criteria and flow rules for porous ductile media. J. Engng. Materials Technol. 1977, 99: 2-15.

9. Tvergaard, V. Influence of voids on shear band instabilities under plane strain conditions. Int. J. Fracture, 1981, 17:389-407.

10. Tvergaard, V., Needleman, A. Analysis of the cup-cone fracture in a round tensile bar. Acta Metall., 1984, 32:157-169.

11. Geltmacher, A.B., Koss, D.A., Matic, P., Stout, M.G. A modeling study of the effect of stress state on void linking during ductile fracture. Acta Materialia, 1996, 44:22012210.

12. Thomson, C.I.A., Worswick, M.J., Pilkey, A.K., Lloyd, D.J., Burger, D. Modeling void nucleation and growth within periodic clusters of particles. J. Mech. Phys. Solids, 1999, 47:1-26.

13. Thomson, C.I.A., Worswick, M.J., Pilkey, A.K., Lloyd, D.J. Void coalescence within periodic clusters of particles. J. Mech. Phys. Solids, 2003, 51:127-146.

14. Bandstra, J.P., Koss, D.A. On the influence of void clusters on void growth and coalescence during ductile fracture. Acta Materialia, 2008, 56:4429-4439. 
15. Babout, L., Maire, E., Fougères, R. Damage initiation in model metallic materials: Xray tomography and modelling. Acta Materialia, 2004, 52:2475-2487.

16. Segurado, J., LLorca, J. Computational micromechanics of composites: The effect of particle spatial distribution. Mechanics of Materials, 2006, 38:873-883.

17. Ohno, N., Hutchinson, J.W. Plastic flow localization due to non-uniform void distribution. J. Mech. Phys. Solids, 1984, 32(1):63-85.

18. Tvergaard, V., Needleman, A. Effect of crack meandering on dynamic, ductile fracture. J. Mech. Phys. Solids, 1992, 40:447-471.

19. Tvergaard, V., Needleman, A. Three dimensional microstructural effects on plane strain ductile crack growth. Int. J. Solids Structures, 2006, 43:6165-6179.

20. Tinet, H., Klöcker, H., Le Coze, J. Damage analysis during hot deformation of a resulfurized stainless steel. Acta Materialia, 2004, 52:3825-3842.

21. Tvergaard, V. Effect of void cluster on ductile failure evolution. Meccanica, 2016, 51:3097-3105.

22. Needleman, A. A continuum model for void nucleation by inclusion debonding. J. Appl. Mech., 1987, 54:525-531.

23. Tvergaard, V. Effect of fibre debonding in a whisker reinforced metal. Mater. Sci. Eng., 1990, A125:203-213.

24. Hutchinson, J.W. Finite strain analysis of elastic-plastic solids and structures. In: Hartung, R.F. (Ed.), Numerical Solution of Nonlinear Structural Problems. ASME, New York, 1973, p. 17.

25. Tvergaard, V. Effect of thickness inhomogeneities in internally pressurized elasticplastic spherical shells. J. Mech. Phys. Solids, 1976, 24:291-304.

26. Tvergaard, V., Hutchinson, J.W. Two mechanisms of ductile fracture: void by void growth versus multiple void interaction. Int. J. Solids Struct., 2002, 39:3581-3597.

27. Tvergaard, V. Discrete modelling of ductile crack growth by void growth to coalescence. Int. J. Fracture, 2007, 148:1-12.

28. Pedersen, T.Ø. Remeshing in analysis of large plastic deformations. Computers and Structures, 1998, 67:279-288.

29. Tvergaard, V. Studies of void growth in a thin ductile layer between ceramics, Computational Mechanics, 1997, 20:186-191.

30. Tvergaard, V. On fatigue crack growth in ductile materials by cracktip blunting. J. Mech. Phys. Solids, 2004, 52:2149-2166. 
31. Needleman, A. A continuum model for void nucleation by inclusion debonding. J. Appl. Mech., 1987, 54:525-531.

32. Tvergaard, V. Effect of fibre debonding in a whisker-reinforced metal. Mater. Sci. and Engng., 1990, A125:203-213.

33. Tvergaard, V., Hutchinson, J.W. Effect of strain-dependent cohesive zone model on predictions of crack growth resistance. 1996, 33:3297-3308. 


\section{Figure captions:}

Fig. 1. Coordinates and dimensions, for a case with $N_{v}=5$. (a) Region near the voids. (b) The unit cell analysed; due to symmetries about the coordinate axes only one quarter of the full cell needs to be analysed numerically.

Fig. 2. Initial mesh near inclusions, for a case with $N_{v}=9$.

Fig. 3. Nucleation parameters $\Sigma_{N} / \sigma_{Y}=3.33$ and $\varepsilon_{N}=0.1$. Curves of normalized stress or void volume vs. logarithmic strain, for the stress ratio $\kappa=0.25$ and for different values $N_{v}$ of the number of inclusions in the cluster. (a) True tensile stress $\Sigma_{22}$ normalized by the initial yield stress. (b) Total void volume $V$ normalized by the initial inclusion volume.

Fig. 4. Deformed mesh for the computation with $N_{v}=9$ in Fig. 3, at $\varepsilon_{2}=0.255$ and $V / V_{0}=1197$.

Fig. 5. Nucleation parameters $\Sigma_{N} / \sigma_{Y}=2.33$ and $\varepsilon_{N}=0.1$. Curves of normalized stress or void volume vs. logarithmic strain, for the stress ratio $\kappa=0.25$ and for different values $N_{v}$ of the number of inclusions in the cluster. (a) True tensile stress $\Sigma_{22}$ normalized by the initial yield stress. (b) Total void volume $V$ normalized by the initial inclusion volume.

Fig. 6. Nucleation parameters $\Sigma_{N} / \sigma_{Y}=2.33$ and $\varepsilon_{N}=0.15$. Curves of normalized stress or void volume vs. logarithmic strain, for the stress ratio $\kappa=0.25$ and for different values $N_{v}$ of the number of inclusions in the cluster. (a) True tensile stress $\Sigma_{22}$ normalized by the initial yield stress. (b) Total void volume $V$ normalized by the initial inclusion volume.

Fig. 7. Nucleation parameters $\Sigma_{N} / \sigma_{Y}=3.33$ and $\varepsilon_{N}=0.1$. Curves of normalized stress or void volume vs. logarithmic strain, for the stress ratio $\kappa=0.40$ and for different values $N_{v}$ of the number of inclusions in the cluster. (a) True tensile stress $\Sigma_{22}$ normalized by the initial yield stress. (b) Total void volume $V$ normalized by the initial inclusion volume.

Fig. 8. Nucleation parameters $\Sigma_{N} / \sigma_{Y}=3.33$ and $\varepsilon_{N}=0.1$. Curves of normalized stress or void volume vs. logarithmic strain, for the stress ratio $\kappa=0.0$ and for different values $N_{v}$ of the number of inclusions in the cluster. (a) True tensile stress $\Sigma_{22}$ normalized by the initial yield stress. (b) Total void volume $V$ normalized by the initial inclusion volume.

Fig. 9. Nucleation parameters $\Sigma_{N} / \sigma_{Y}=6.67$ and $\varepsilon_{N}=0.3$. Curves of normalized stress or void volume vs. logarithmic strain, for the stress ratio $\kappa=0.25$ and for different values $N_{v}$ of the number of inclusions in the cluster. (a) True tensile stress $\Sigma_{22}$ normalized by the initial yield stress. (b) Total void volume $V$ normalized by the initial inclusion volume.

Fig. 10. Deformed mesh for the computation with $N_{v}=9$ in Fig. 9, at $\varepsilon_{2}=0.395$ and $V / V_{0}=1197$. 
Fig. 11. Nucleation parameters $\Sigma_{N} / \sigma_{Y}=3.33$ and $\varepsilon_{N}=0.1$, while the power hardening exponent is $n=5$. Curves of normalized stress or void volume vs. logarithmic strain, for the stress ratio $\kappa=0.25$ and for different values $N_{v}$ of the number of inclusions in the cluster. (a) True tensile stress $\Sigma_{22}$ normalized by the initial yield stress. (b) Total void volume $V$ normalized by the initial inclusion volume.

Fig. 12. Nucleation parameters $\Sigma_{N} / \sigma_{Y}=3.33$ and $\varepsilon_{N}=0.1$, while the power hardening exponent is $n=20$. Curves of normalized stress or void volume vs. logarithmic strain, for the stress ratio $\kappa=0.25$ and for different values $N_{v}$ of the number of inclusions in the cluster. (a) True tensile stress $\Sigma_{22}$ normalized by the initial yield stress. (b) Total void volume $V$ normalized by the initial inclusion volume. 


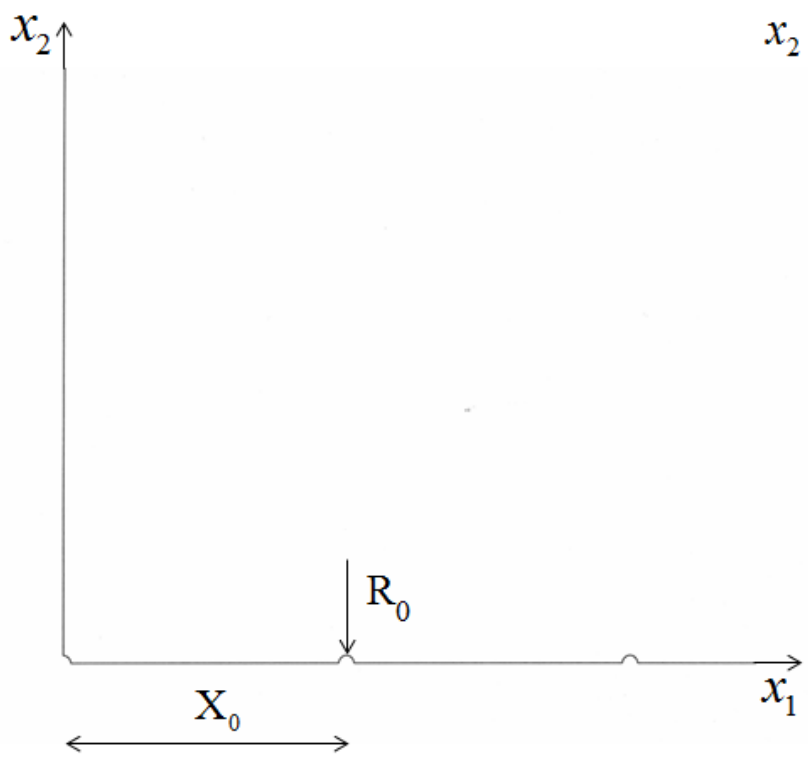

(a)

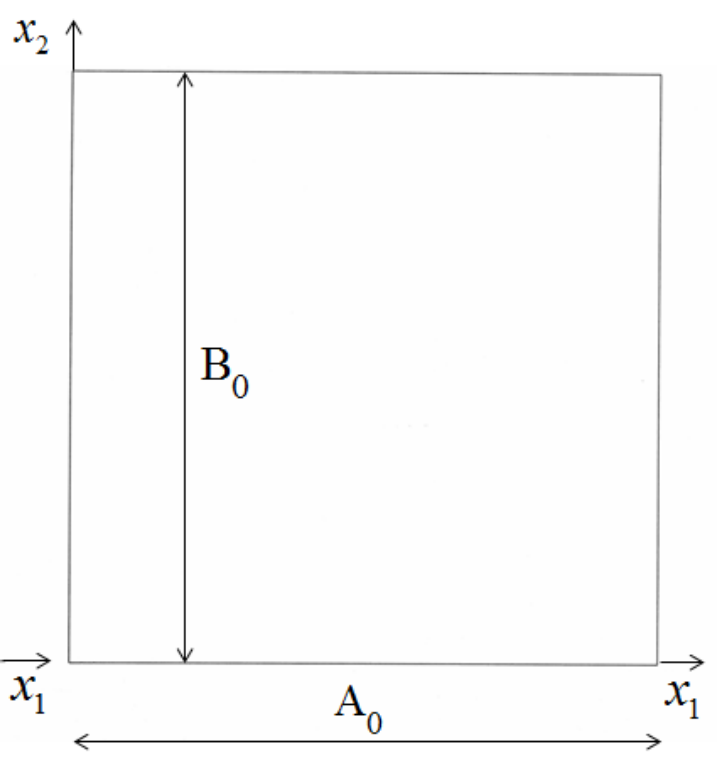

(b)

Fig. 1. Coordinates and dimensions, for a case with $N_{v}=5$. (a) Region near the voids. (b) The unit cell analysed; due to symmetries about the coordinate axes only one quarter of the full cell needs to be analysed numerically.

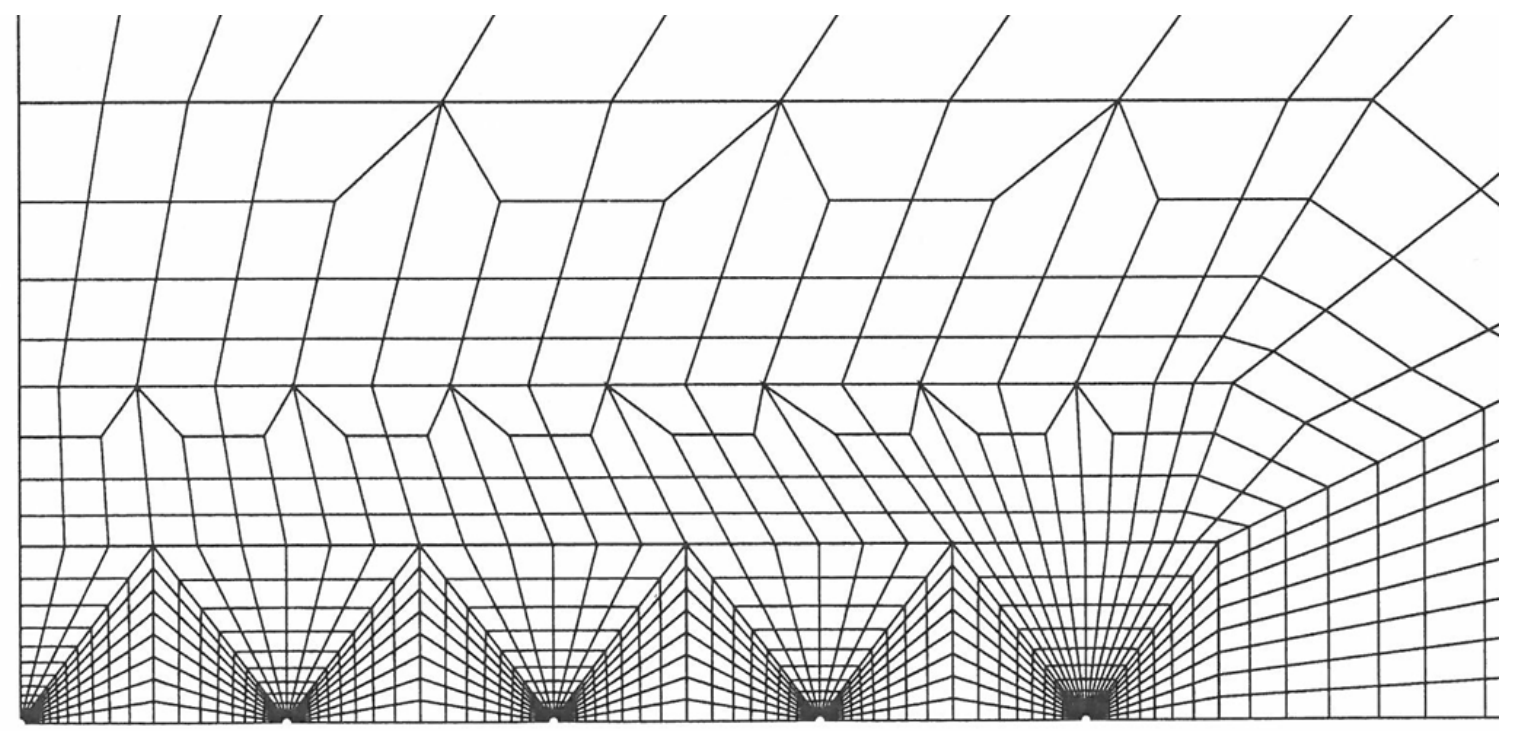

Fig. 2. Initial mesh near inclusions, for a case with $N_{v}=9$. 

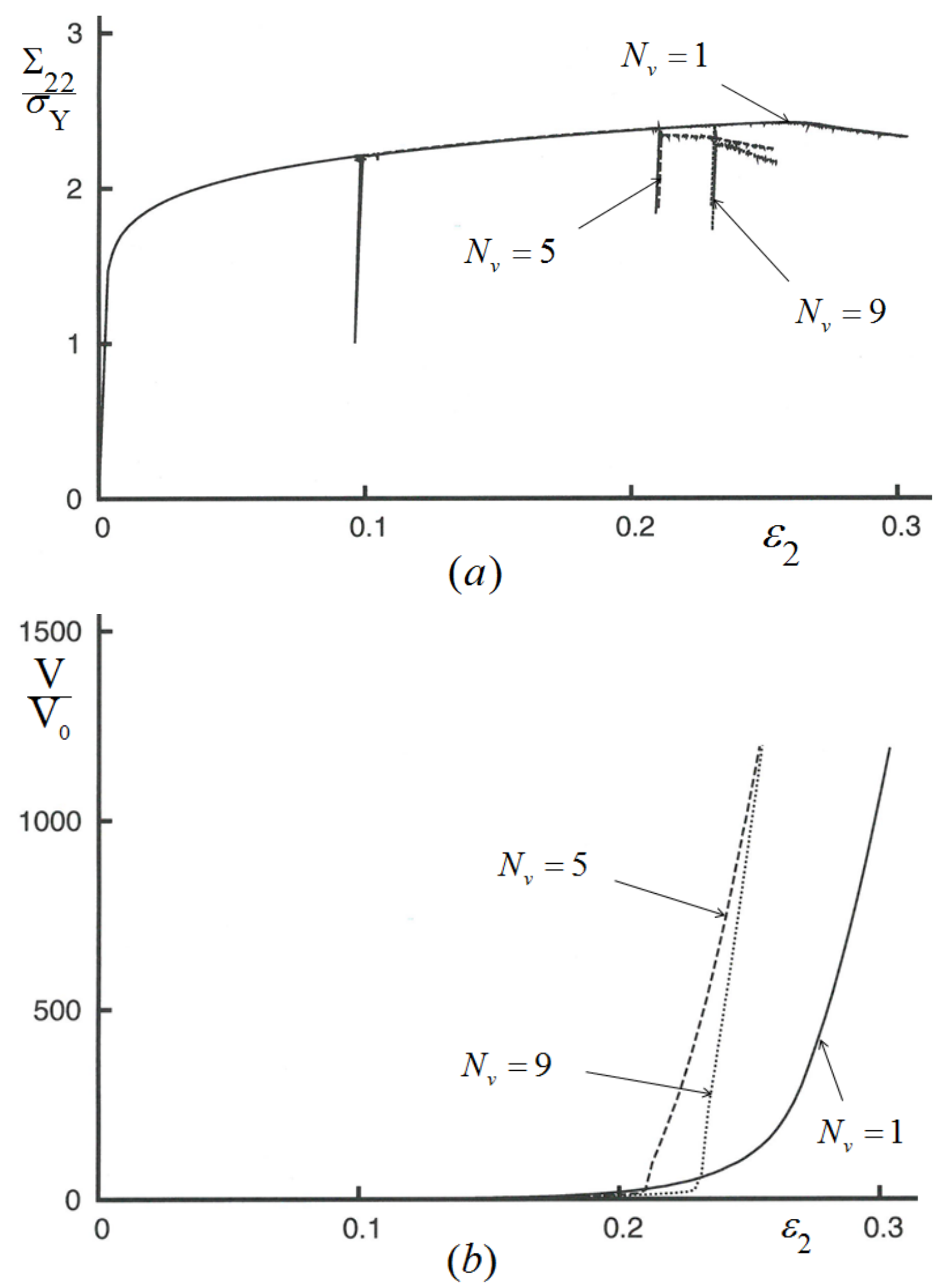

Fig. 3. Nucleation parameters $\Sigma_{N} / \sigma_{Y}=3.33$ and $\varepsilon_{N}=0.1$. Curves of normalized stress or void volume vs. logarithmic strain, for the stress ratio $\kappa=0.25$ and for different values $N_{v}$ of the number of inclusions in the cluster. (a) True tensile stress $\Sigma_{22}$ normalized by the initial yield stress. (b) Total void volume $V$ normalized by the initial inclusion volume. 


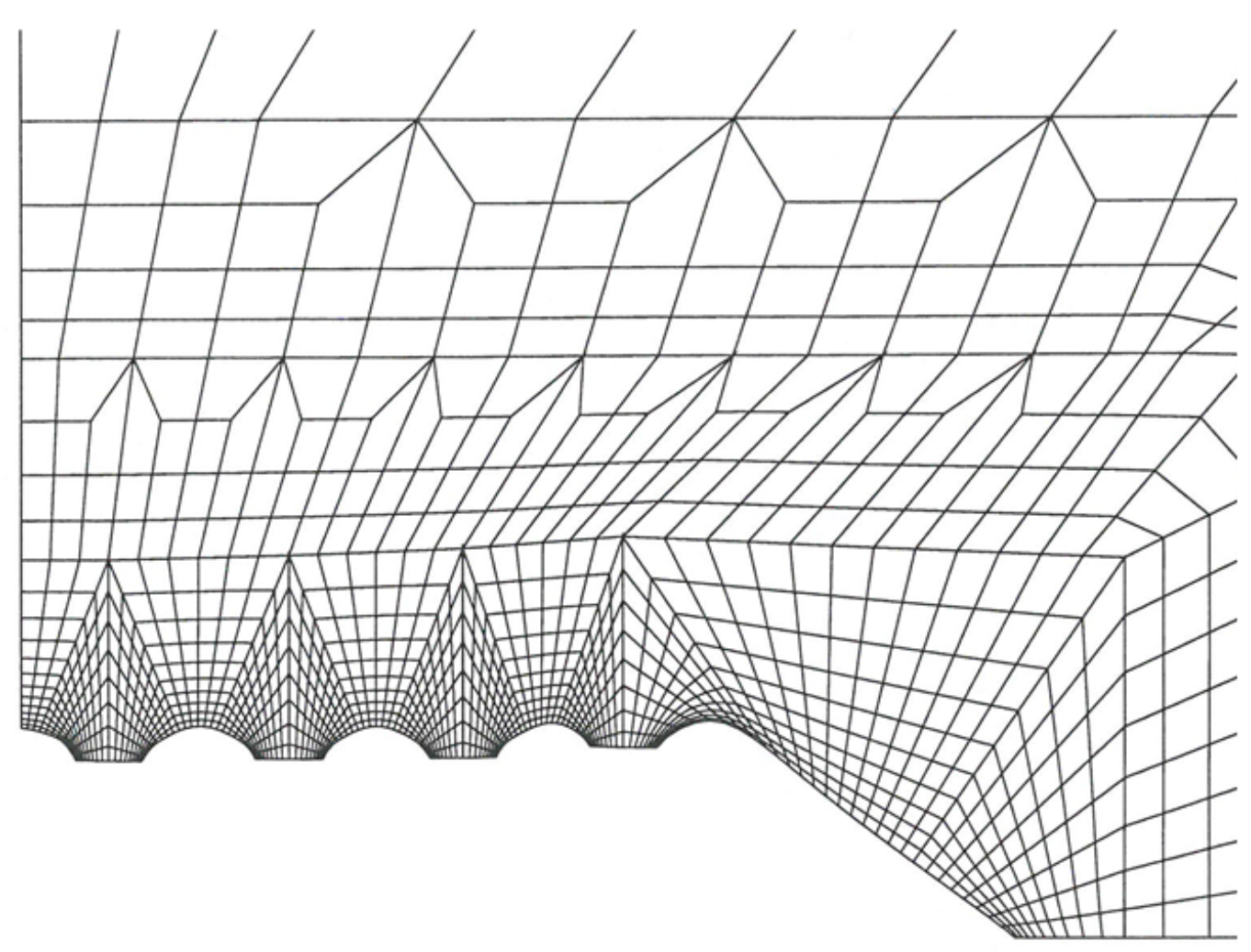

Fig. 4. Deformed mesh for the computation with $N_{v}=9$ in Fig. 3, at $\varepsilon_{2}=0.255$ and $V / V_{0}=1197$. 

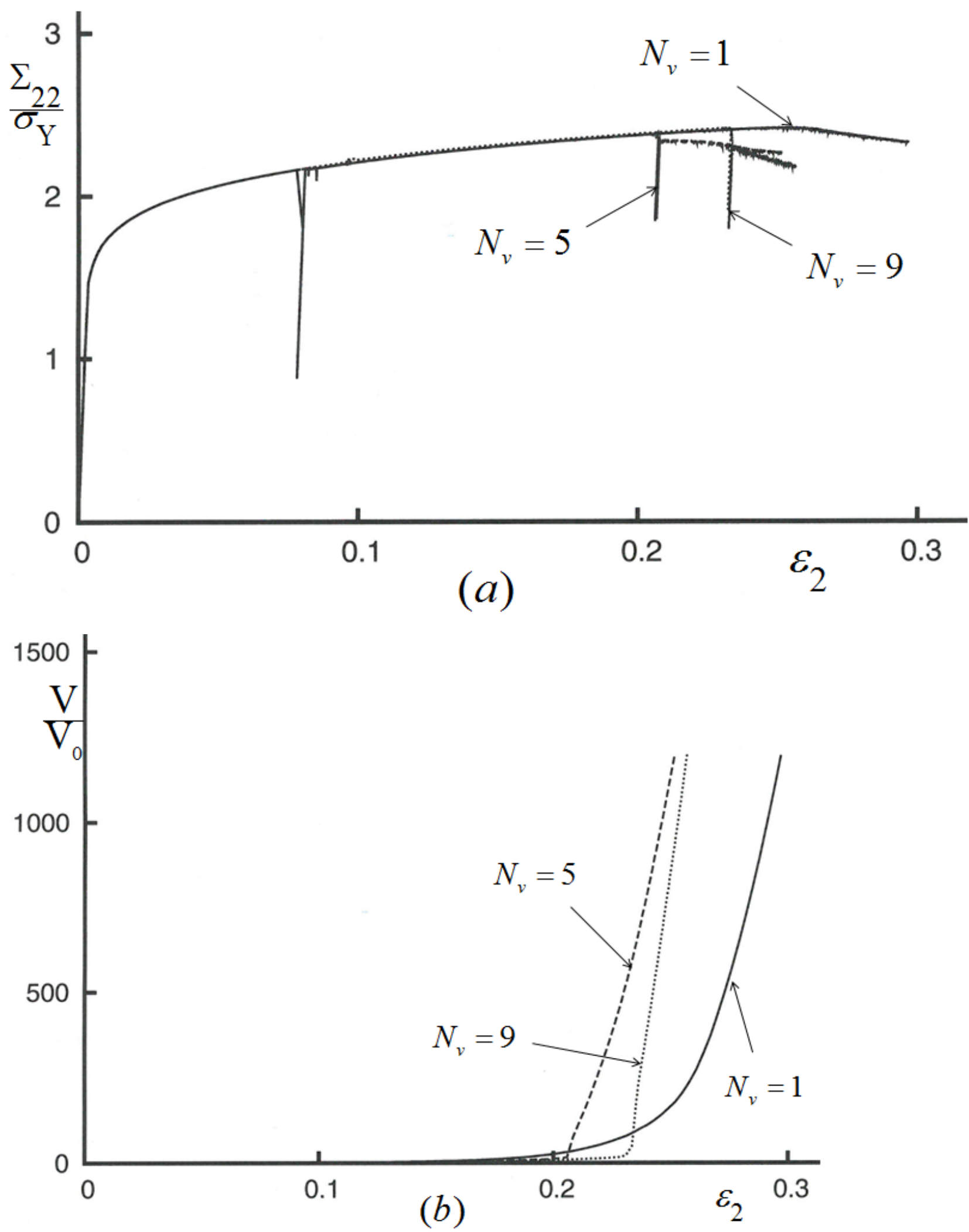

Fig. 5. Nucleation parameters $\Sigma_{N} / \sigma_{Y}=2.33$ and $\varepsilon_{N}=0.1$. Curves of normalized stress or void volume vs. logarithmic strain, for the stress ratio $\kappa=0.25$ and for different values $N_{v}$ of the number of inclusions in the cluster. (a) True tensile stress $\Sigma_{22}$ normalized by the initial yield stress. (b) Total void volume $V$ normalized by the initial inclusion volume. 

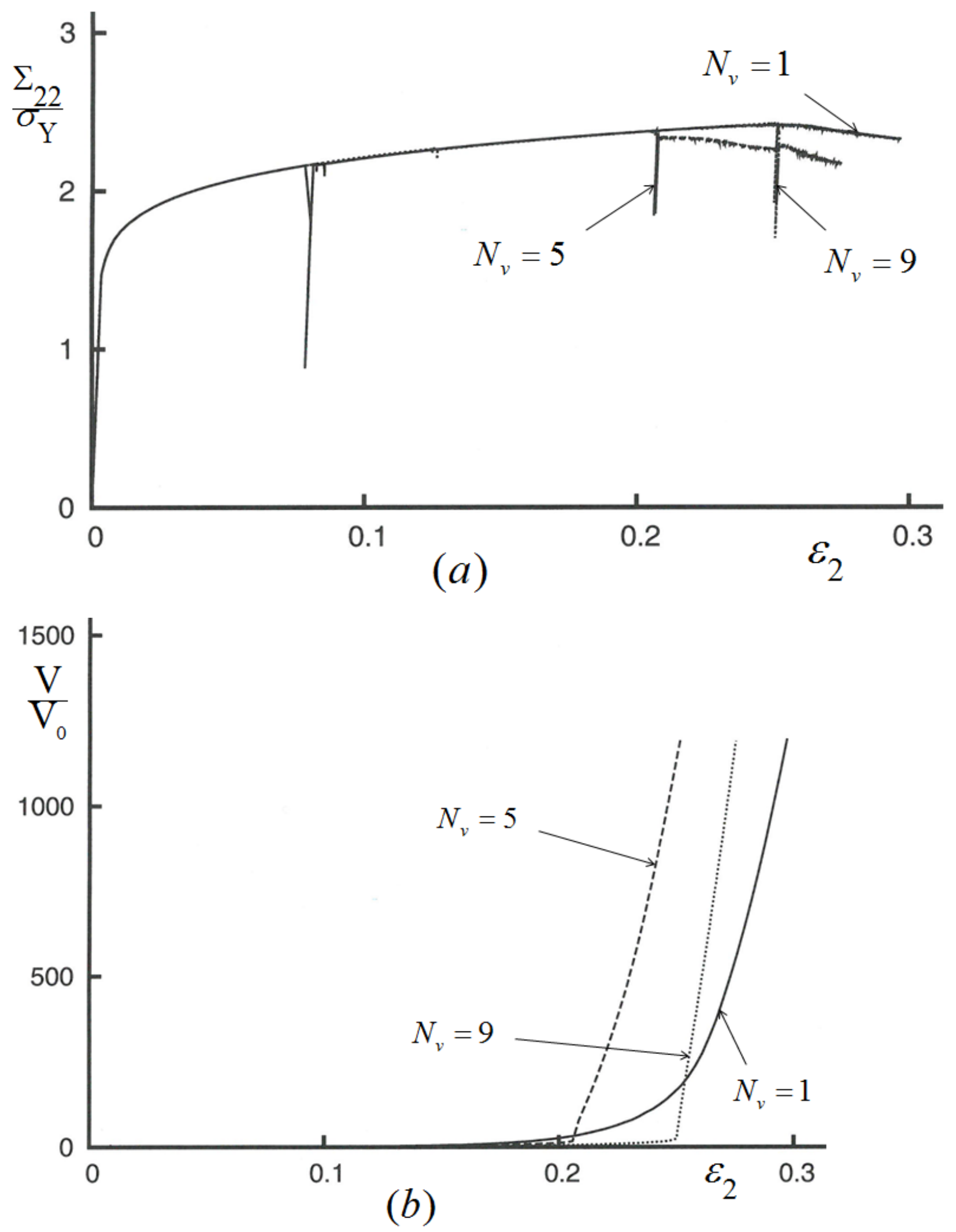

Fig. 6. Nucleation parameters $\Sigma_{N} / \sigma_{Y}=2.33$ and $\varepsilon_{N}=0.15$. Curves of normalized stress or void volume vs. logarithmic strain, for the stress ratio $\kappa=0.25$ and for different values $N_{v}$ of the number of inclusions in the 
cluster. (a) True tensile stress $\Sigma_{22}$ normalized by the initial yield stress. (b) Total void volume $V$ normalized by the initial inclusion volume.
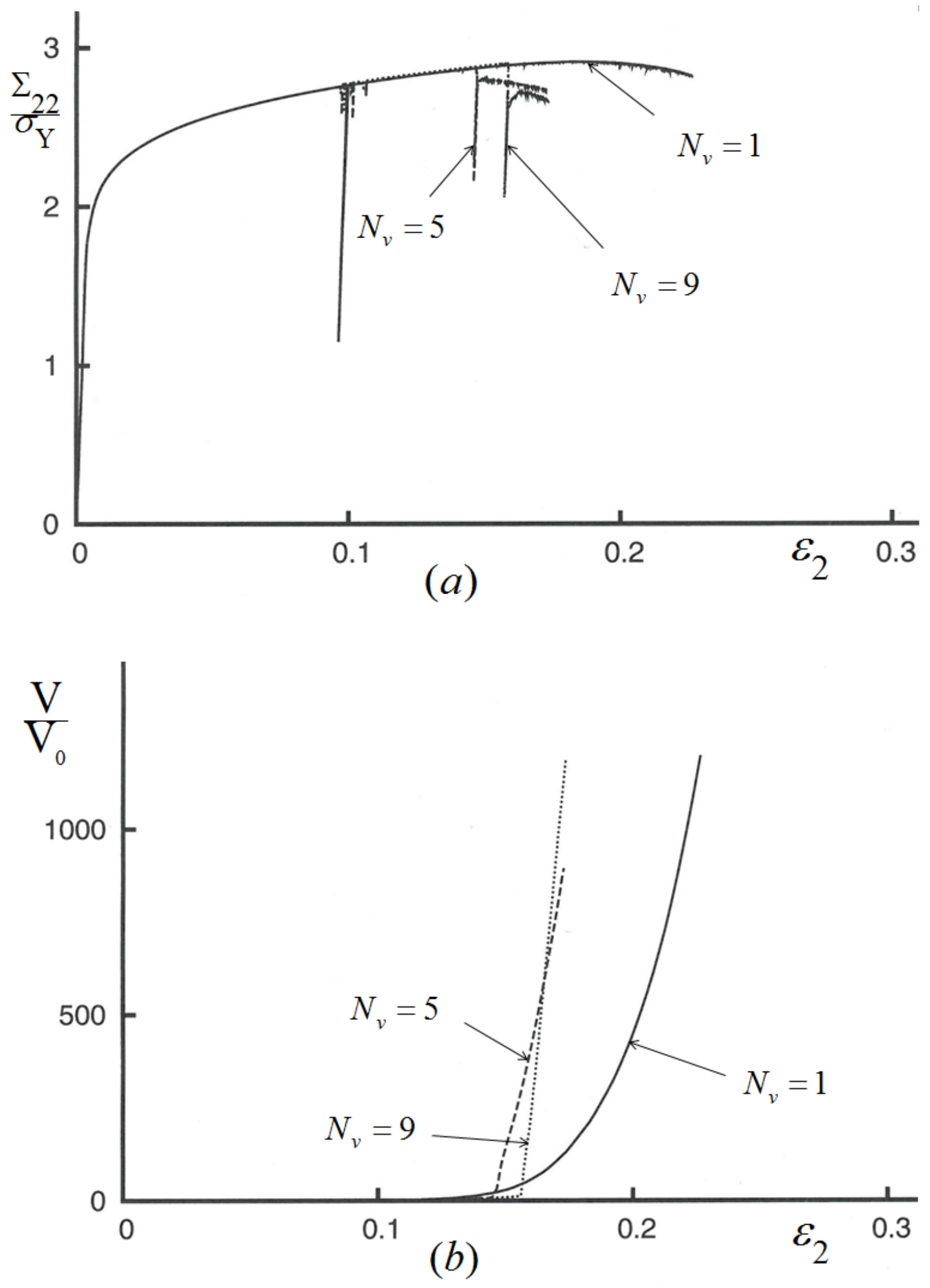
Fig. 7. Nucleation parameters $\Sigma_{N} / \sigma_{Y}=3.33$ and $\varepsilon_{N}=0.1$. Curves of normalized stress or void volume vs. logarithmic strain, for the stress ratio $\kappa=0.40$ and for different values $N_{v}$ of the number of inclusions in the cluster. (a) True tensile stress $\Sigma_{22}$ normalized by the initial yield stress. (b) Total void volume $V$ normalized by the initial inclusion volume.

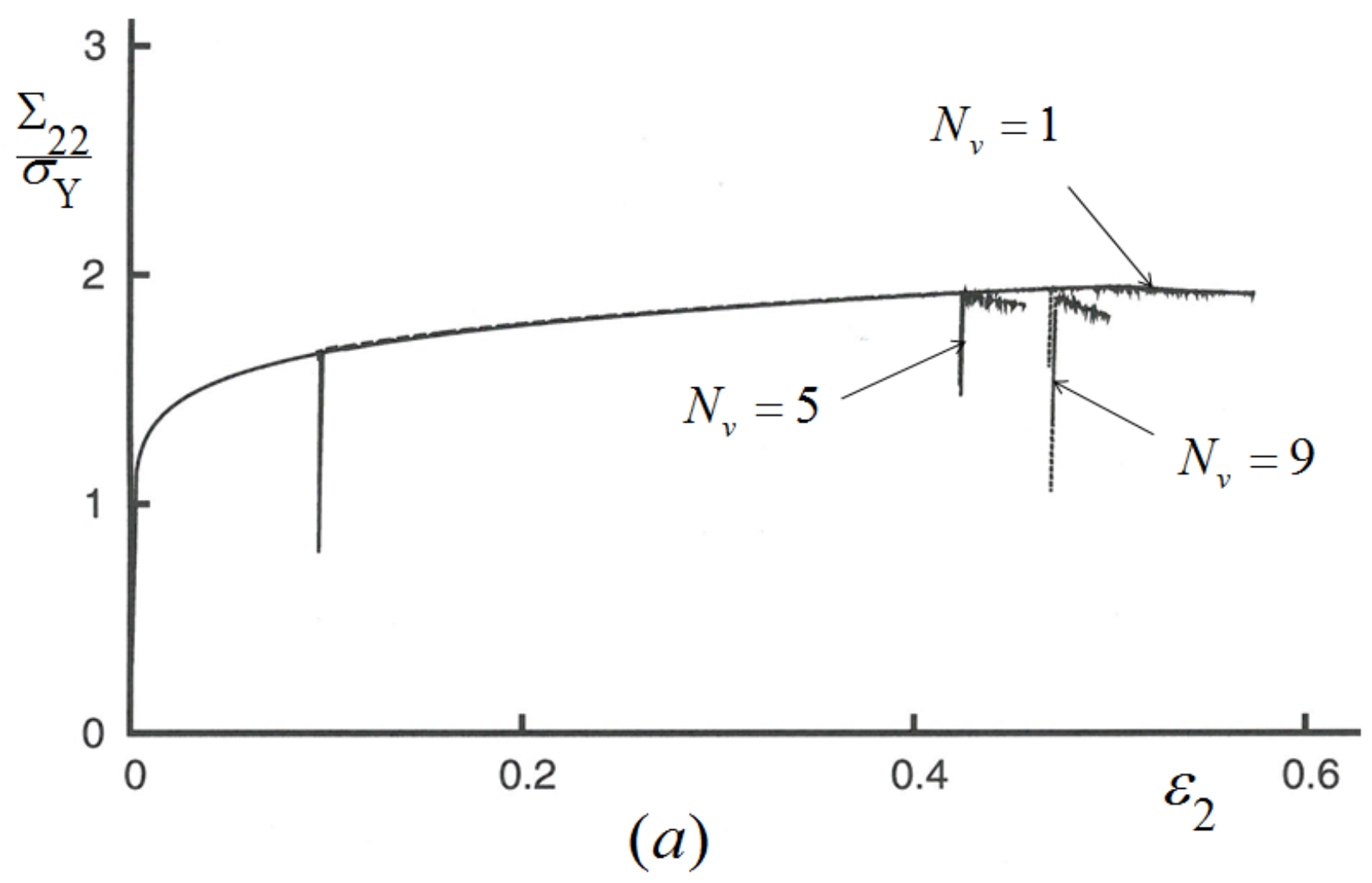




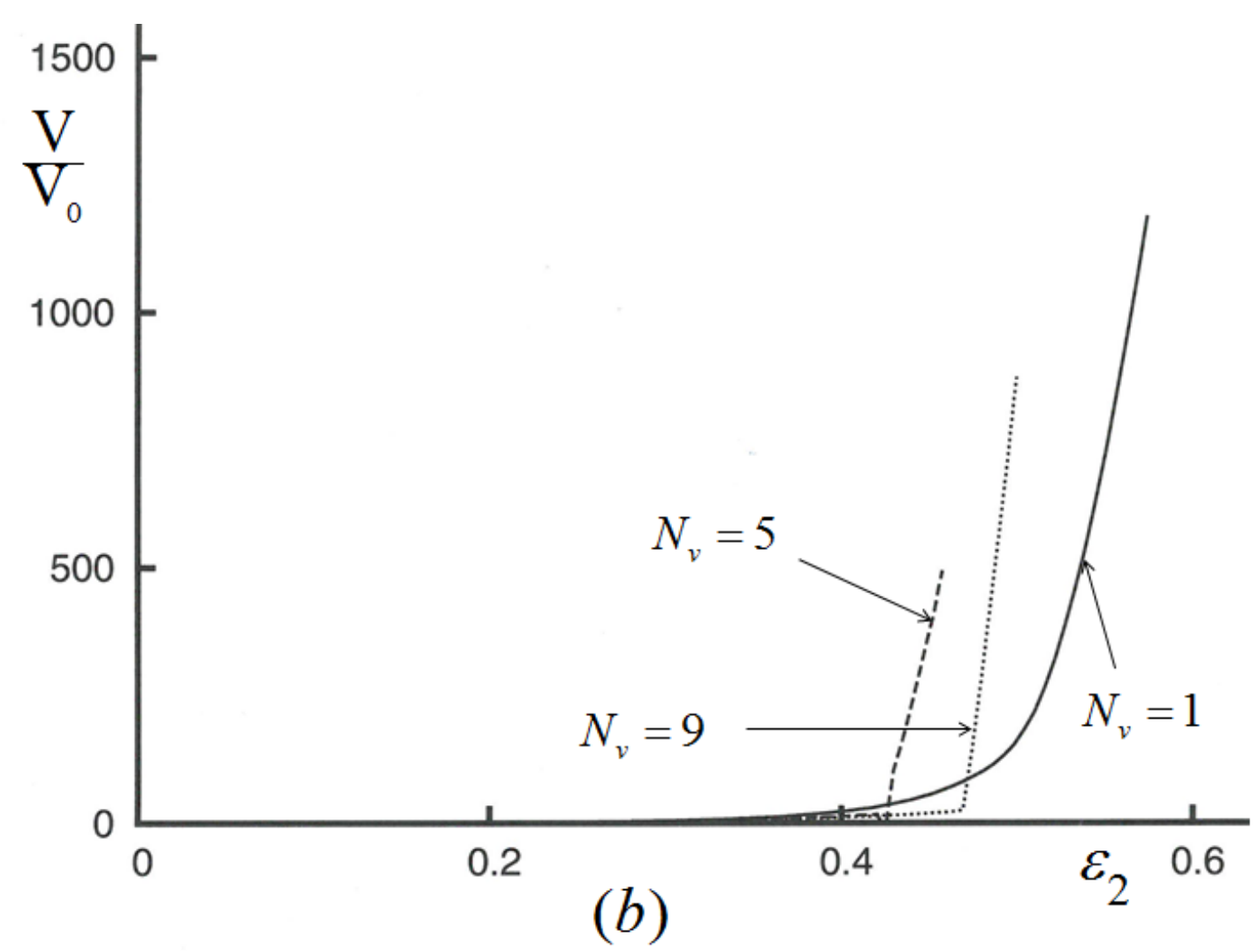

Fig. 8. Nucleation parameters $\Sigma_{N} / \sigma_{Y}=3.33$ and $\varepsilon_{N}=0.1$. Curves of normalized stress or void volume vs. logarithmic strain, for the stress ratio $\kappa=0.0$ and for different values $N_{v}$ of the number of inclusions in the cluster. (a) True tensile stress $\Sigma_{22}$ normalized by the initial yield stress. (b) Total void volume $V$ normalized by the initial inclusion volume. 

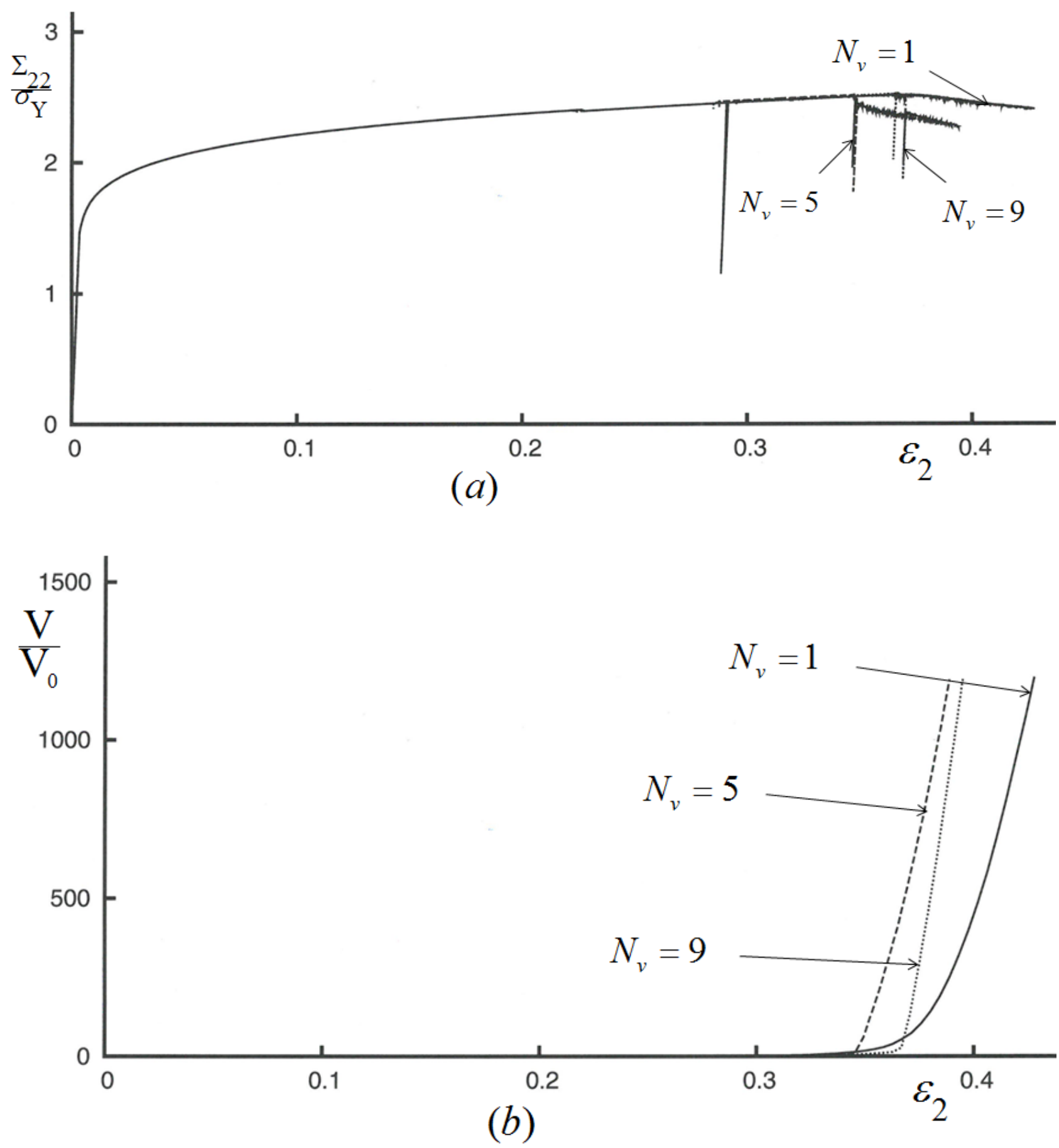

Fig. 9. Nucleation parameters $\Sigma_{N} / \sigma_{Y}=6.67$ and $\varepsilon_{N}=0.3$. Curves of normalized stress or void volume vs. logarithmic strain, for the stress ratio $\kappa=0.25$ and for different values $N_{v}$ of the number of inclusions in the cluster. (a) True tensile stress $\Sigma_{22}$ normalized by the initial yield stress. (b) Total void volume $V$ normalized by the initial inclusion volume. 


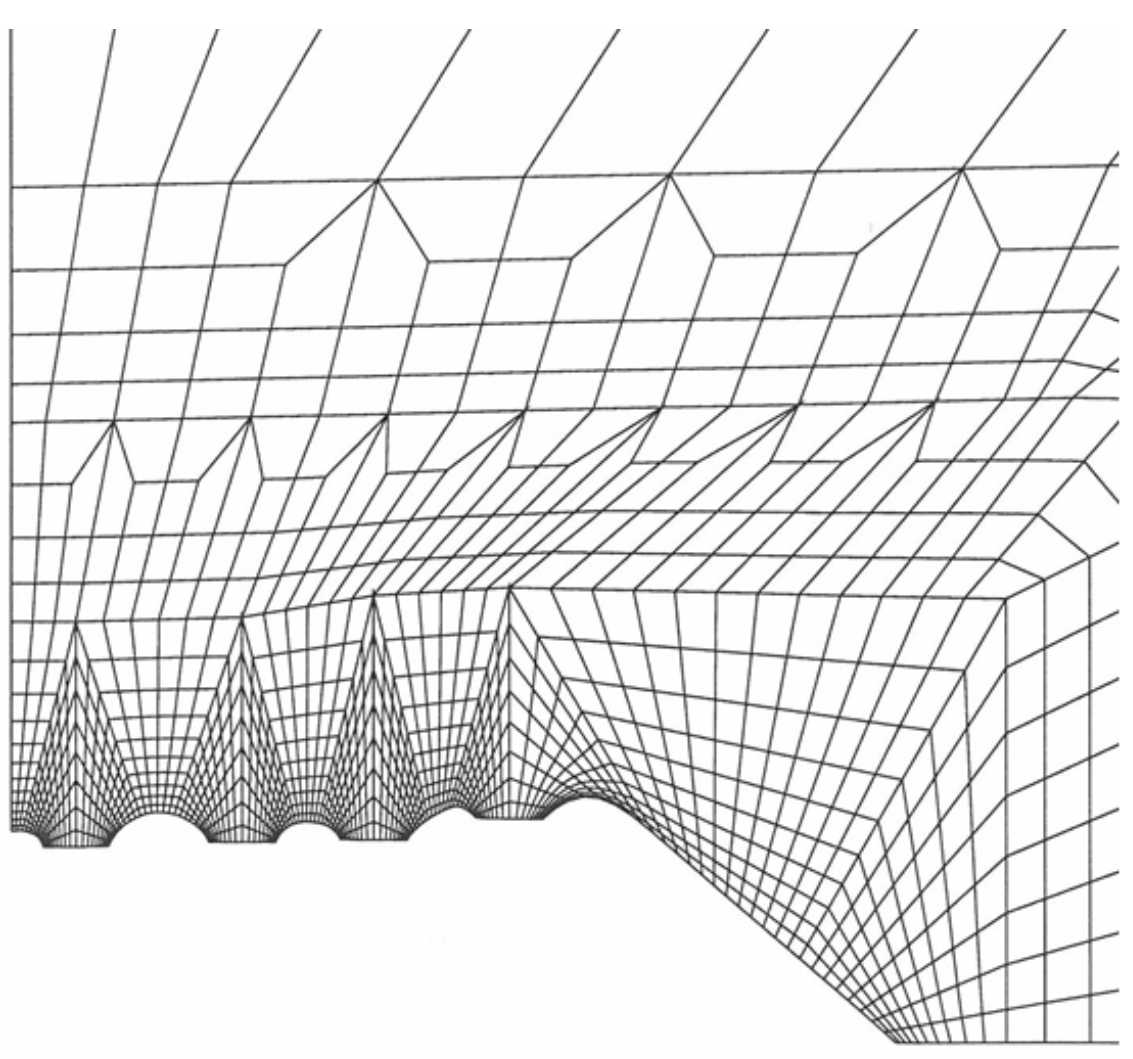

Fig. 10. Deformed mesh for the computation with $N_{v}=9$ in Fig. 9, at $\varepsilon_{2}=0.395$ and $V / V_{0}=1197$.

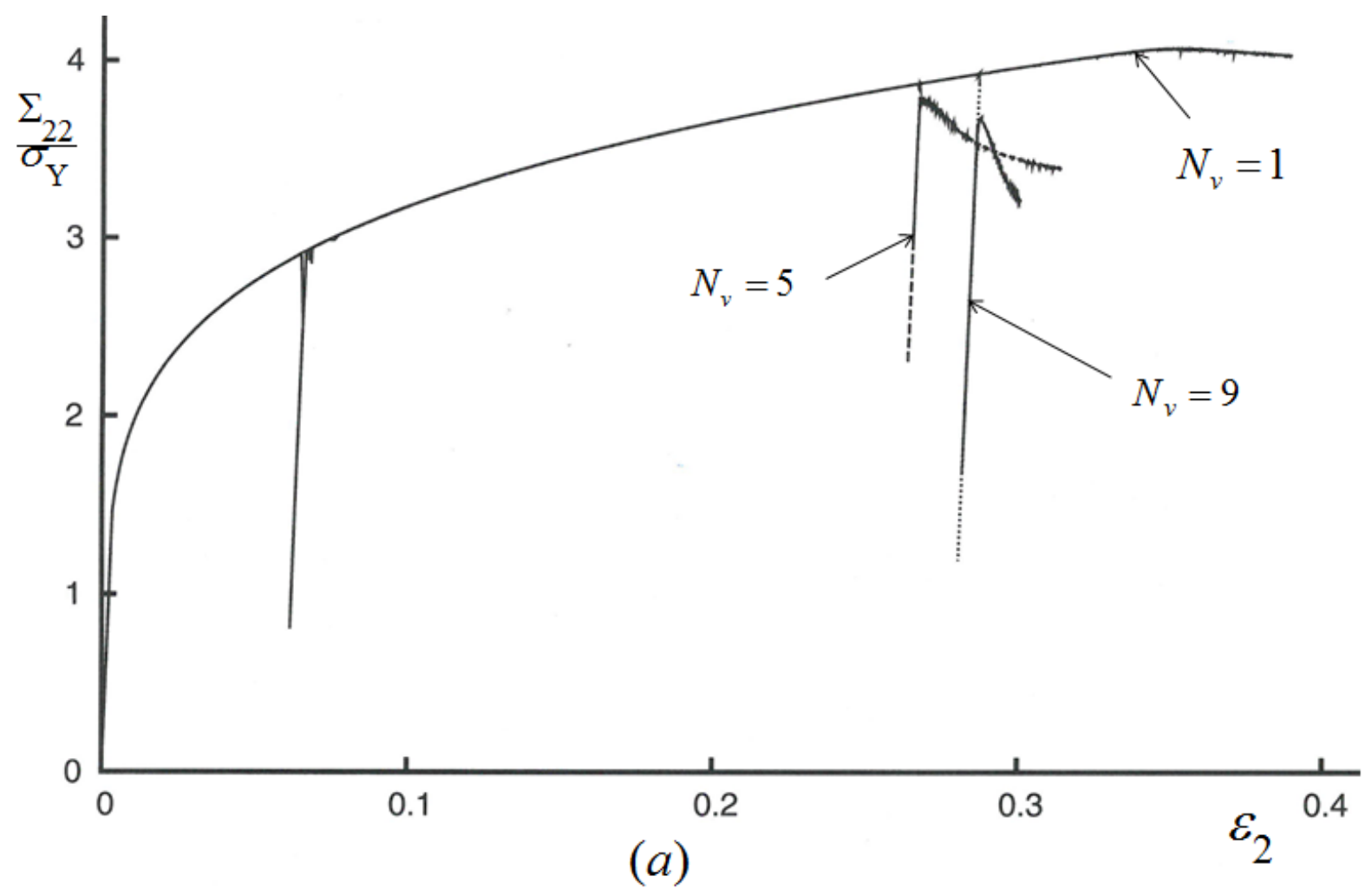




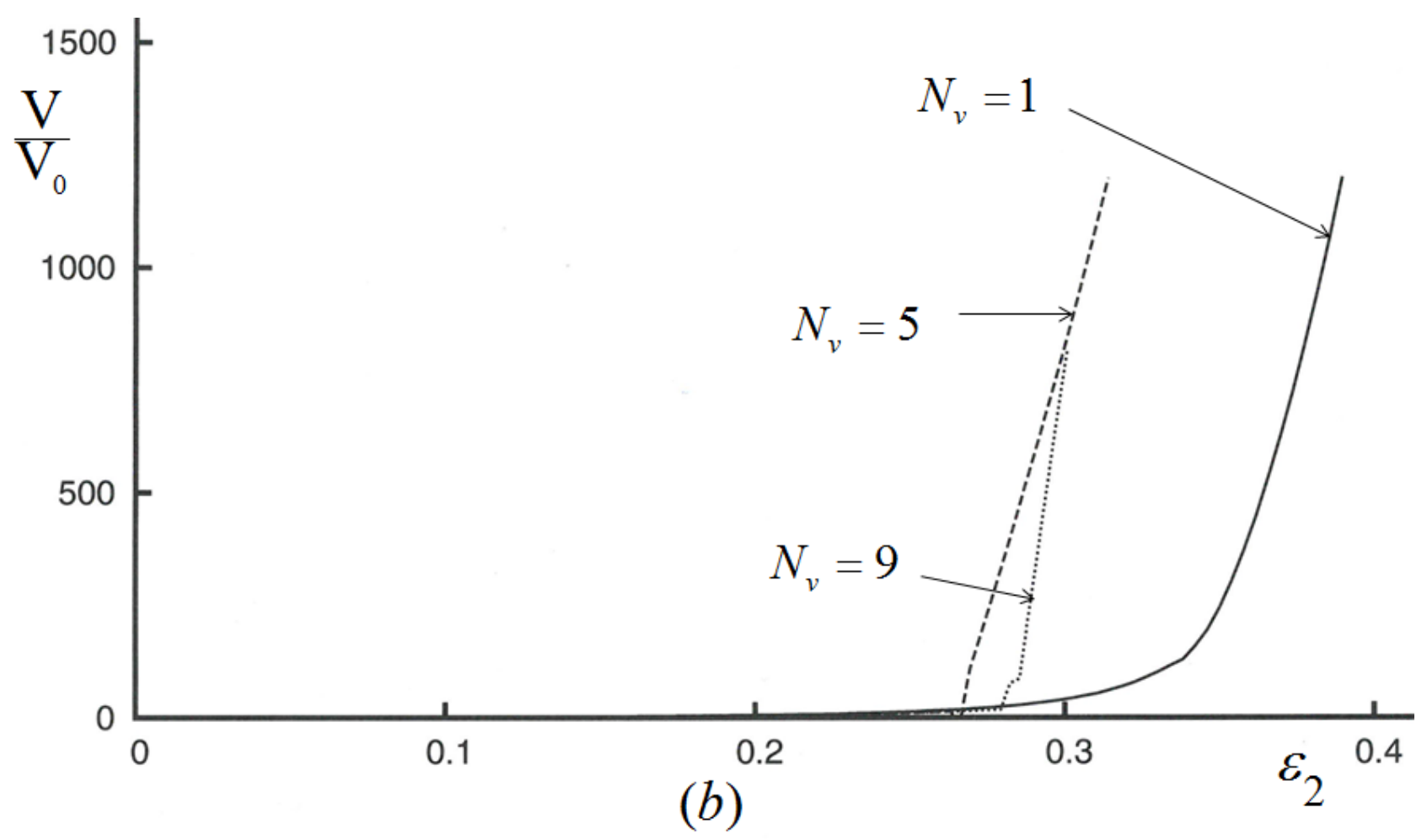

Fig. 11. Nucleation parameters $\Sigma_{N} / \sigma_{Y}=3.33$ and $\varepsilon_{N}=0.1$, while the power hardening exponent is $n=5$. Curves of normalized stress or void volume vs. logarithmic strain, for the stress ratio $\kappa=0.25$ and for different values $N_{v}$ of the number of inclusions in the cluster. (a) True tensile stress $\Sigma_{22}$ normalized by the initial yield stress. (b) Total void volume $V$ normalized by the initial inclusion volume.

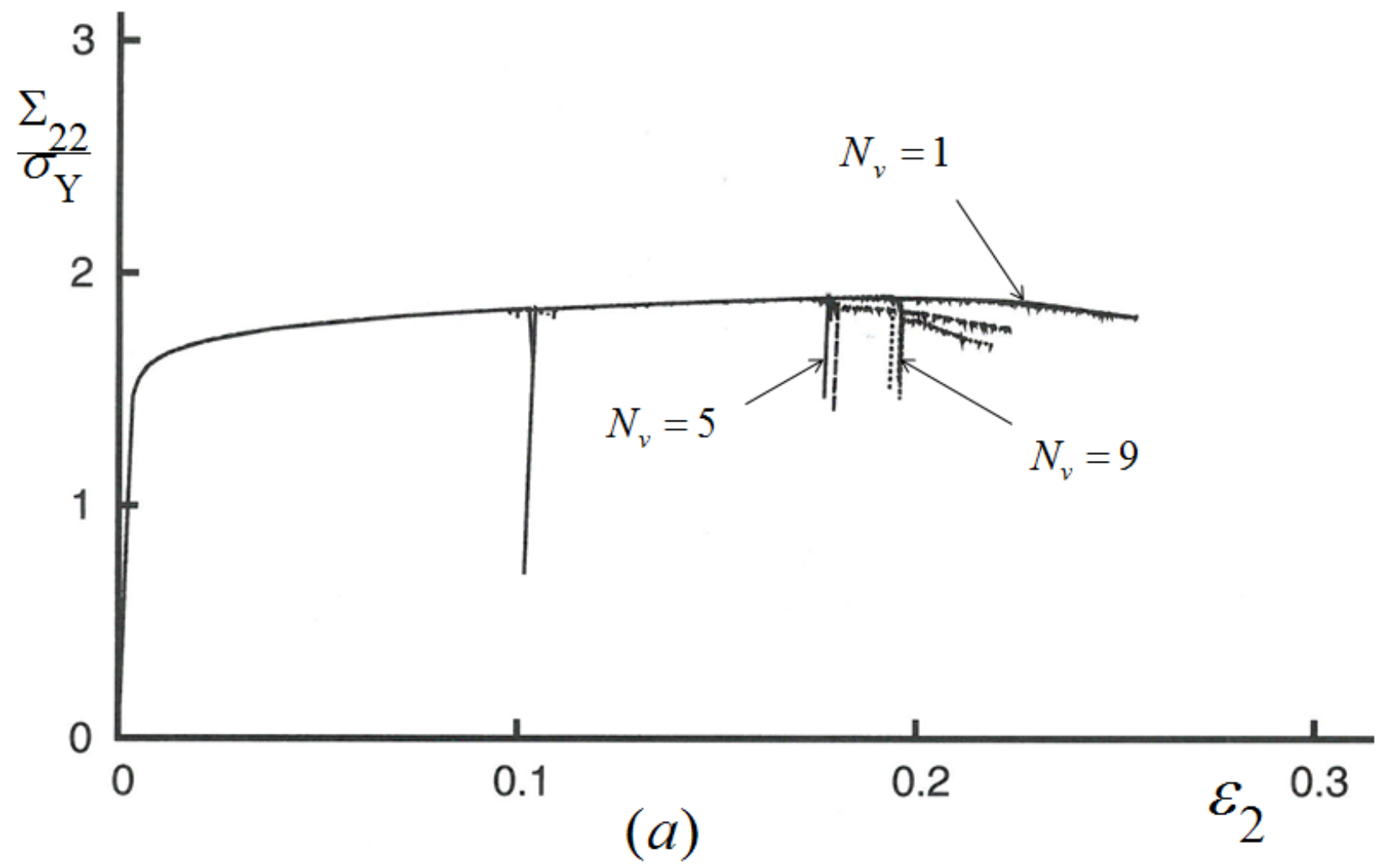




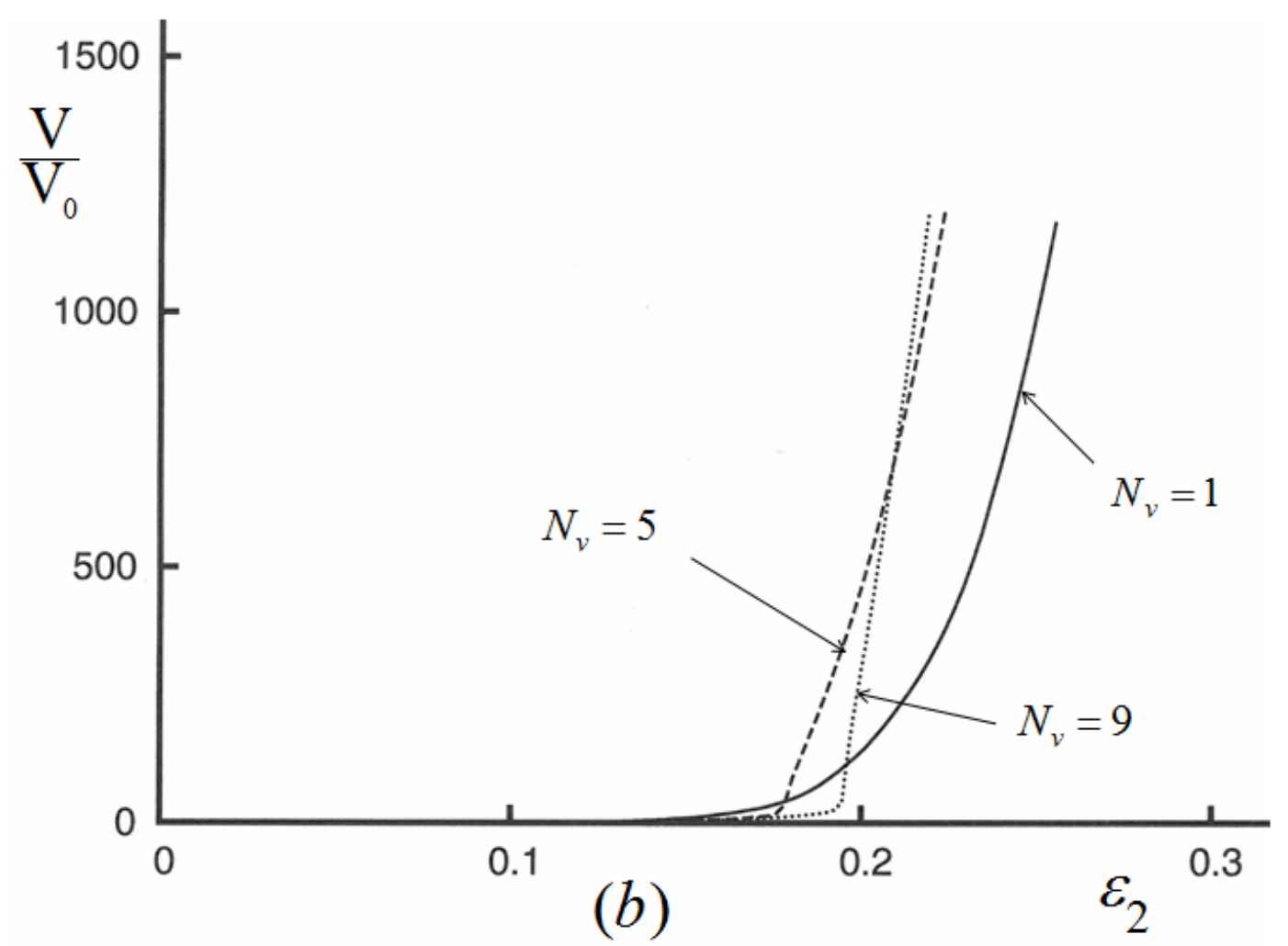

Fig. 12. Nucleation parameters $\Sigma_{N} / \sigma_{Y}=3.33$ and $\varepsilon_{N}=0.1$, while the power hardening exponent is $n=20$. Curves of normalized stress or void volume vs. logarithmic strain, for the stress ratio $\kappa=0.25$ and for different values $N_{v}$ of the number of inclusions in the cluster. (a) True tensile stress $\Sigma_{22}$ normalized by the initial yield stress. (b) Total void volume $V$ normalized by the initial inclusion volume. 